\title{
ESCRTs in membrane sealing
}

Maja Radulovic ${ }^{1,2}$ and Harald Stenmark ${ }^{1,2}$

${ }^{1}$ Department of Molecular Cell Biology, Institute for Cancer Research, Oslo University Hospital, Montebello, N-0379 Oslo, Norway

${ }^{2}$ Centre for Cancer Cell Reprogramming, Faculty of Medicine, University of Oslo, Montebello, N-0379 Oslo, Norway

Correspondence: stenmark@ulrik.uio.no, telephone +47 22781818, fax +47 22781845 


\begin{abstract}
The multi-subunit endosomal sorting complex required for transport (ESCRT) machinery is a key regulator of cellular membrane dynamics. Initially characterized in the budding yeast Saccharomyces cerevisiae for its involvement in cargo sorting to the vacuole, the yeast lysosome, this protein complex has emerged over the past decade as a driver for diverse membrane remodeling processes. Their pleiotropic functional connection is mirrored in numerous cellular processes such as cytokinetic abscission during the final step of cell division, nuclear pore quality control, nuclear envelope sealing and repair, plasma membrane repair, vesicle shedding from the plasma membrane, viral budding, and axonal pruning. Common to all the processes regulated by the ESCRT machinery is their assembly on the cytosolic side of the respective membrane to stabilize concave membranes, budding, and scission of narrow membrane necks away from the cytosol. Thus, this machinery has evolved to perform a number of functions in membrane dynamics, and given its importance it is not surprising that dysfunctional ESCRT machinery has been implicated in several diseases. In this mini-review we summarize the role of ESCRT proteins in membrane deformation specifically during membrane sealing and repair.
\end{abstract}




\section{Introduction}

The evolutionarily conserved endosomal sorting complex required for transport (ESCRT) proteins were originally identified in a yeast genetic screen as class $E$ vps (vacuolar protein sorting) mutants due to endosomal accumulation and missorting of vacuolar proteins (CPY and V-ATPase), and were named after their role in sorting of membrane proteins from endosomes to lysosomes by mediating the formation of multivesicular endosomes (MVEs) $[1,2]$. The intense research that followed their initial discovery has uncovered that the ESCRT machinery constitutes four multimeric protein core complexes termed ESCRT-0, ESCRT-I, ESCRT-II, ESCRT-III plus the VPS4 ATPase complex and additional accessory proteins such as Bro1 protein family members. Normally localized in the cytoplasm, ESCRTs get sequentially recruited by different classes of adaptor proteins to different membranes.

It is now clear that recruitment of ESCRT proteins to various membranes is a finely tuned process, which involves distinct mechanisms. For instance, the ESCRT-0 complex is crucial for consecutive recruitment of other ESCRT components to endosomes, CEP55 mediates recruitment of ESCRT-I and the Bro-domain protein ALIX to intercellular bridges to facilitate cytokinetic abscission, and CHMP7 recruits ESCRT-III to the reforming or ruptured nuclear envelope [3-6]. The human immunodeficiency virus-1 structural protein Gag employs late assembly domains to bind the ESCRT-I subunit via its ubiquitin enzyme variant domain to promote virus budding from the plasma membrane [7-10]. Therefore, not all ESCRTmediated biological processes require all complexes, but ESCRT-III and VPS4 appear to be universally required.

In mammalian cells, the ESCRT-0 complex comprises HRS (hepatocyte growth factorregulated tyrosine kinase substrate) and STAM (signal transducing adaptor molecule) which interact in an equimolar ratio via coiled-coil GAT domains. Besides two intertwined GAT domains, an amino-terminal VHS domain, ubiquitin- and clathrin- binding domains, another characteristic feature of the HRS subunit is its ability to bind the endosomal lipid phosphatidylinositol 3-phosphate (Ptdlns(3)P) through its FYVE zinc-finger domain [11, 12]. The interaction of the Hrs subunit with Ptdlns(3)P triggers ESCRT recruitment to endosomes [13]. PtdIns(3)P bound HRS recruits the ESCRT-I complex via binding to TSG101 (tumor susceptibility gene 101), a subunit of ESCRT-I to initiate the assembly of the other ESCRTs $[3,14]$. The ESCRT-I complex comprises four subunits TSG101, VPS28, VPS37, MVB12 (multivesicular sorting factor 12) or Ubap1 (ubiquitin-associated protein 1), which are able to interact with both ESCRT-0 and ESCRT-II at the opposite ends of the complex. Via its carboxy-terminal domain, VPS28 interacts with a stable heterotetrameric complex ESCRT-II, by binding to the subunit EAP45 (ELL-associated protein 45). Finally, the subunits of the ESCRT-III complex, the CHMP proteins (charged multivesicular body proteins, or chromatin modifying proteins) such as CHMP2, CHMP3, CHMP4, CHMP6 and their isoforms, are assembled to form the central hub for membrane scission.

Among the CHMP proteins, CHMP6, which nucleates ESCRT-III assembly on membranes, is thought to interact directly with membranes via myristoylation. In addition, another CHMP protein, CHMP4B, can bind to accessory Bro1 domain proteins such as ALIX (ALG-2 interacting protein X; ALG-2: apoptosis-linked gene 2), HD-PTP (His-domain protein tyrosine pseudophosphatase) and BROX [6, 15-18]. CHMP4B is the most abundant of the CHMP proteins, but all CHMPs share the common feature that they are soluble in the cytosol in their closed, autoinhibited stage, and polymerize upon activation and conformational change on 
membranes. However, the exact mechanism on how ESCRT-III filaments sculpt, constrict and cut membranes in vivo is unclear. Nonetheless, ESCRT-IIl's ability to sever membrane necks of different sizes is striking. For example, in mammalian cells the intraluminal vesicles (ILVs) in MVEs have a diameter of $50 \mathrm{~nm}$, while the cytokinetic bridge is constricted to up to $200 \mathrm{~nm}$ prior to the abscission [19-21]. Finally, membrane constriction and the dissociation of ESCRT complexes from membranes is catalyzed by the ATPase VPS4. Recent reports have revealed the ability of membrane-recruited ESCRT-III subunits to constrict and sever narrow membrane necks not only at the endosomes but also at the cytoplasmic side of other cellular membranes that undergo bending and deformation such as the plasma membrane and nuclear envelope.

\section{ESCRTs in membrane remodeling: new pits old fix}

Classical COP-I, COP-II, and clathrin-mediated budding events involve vesicle budding into the cytoplasm. Briefly, coat proteins encapsulate the newly forming vesicle, whereby adaptor proteins help to sequester cargo and promote vesicle maturation and a scission process that liberates the vesicle from the source membrane. The ESCRT machinery is unique in that it creates "inverse" membrane deformation, which is topologically consistent between the ESCRT-dependent processes, yet mechanistically distinct from classical membrane budding. It results in membrane curvature that pushes away from the cell cytoplasm, without providing a coat around the forming membrane bud. Therefore, it is of fundamental importance to understand how the ESCRT machinery draws the opposing membranes together and mediates fission. The subcomplex that is most directly involved in membrane reshaping is ESCRT-III with its subunits visualized by electron microscopy to polymerize into many different forms such as spirals, tubes, coils and cones [22-24]. Its capability of polymerizing into different structures further points to the complexity of the remodeling process and various roles at the different steps.

Recently, in vitro reconstitution systems have allowed for measuring the kinetics of ESCRT assembly $[25,26]$. These analyses have shown that nucleation of CHMP4B filaments occurs faster on negatively curved membranes than on flat membranes and is accelerated by the presence of ESCRT-II and CHMP6 proteins. On the other hand, the kinetics of CHMP4B polymerization is independent of membrane curvature and upstream targeting factors. Thus far, the precise scission mechanism is unclear. There are several models suggested so far, e.g. dome, reverse dome and buckling models [17, 22, 27]. Briefly, the dome model holds that ESCRT-III filaments spiral inward while forming consecutively narrow rings. The spiraling dome model is attractive since ESCRT-III assemblies could initially form rings of different sizes. Here, VPS4 could help further contracting the polymers or even help during the hemifission to fission transition [16, 28, 29]. The reverse dome model describes the same type of constrictions but from the opposite side. The role of VPS4 could be a remodeling of the ESCRT-III assembly as it would have to invert eventually. Finally, in the third model nucleation happens at the negative membrane curvature and filaments then grow outwards from the narrowest point towards the center. Of note, ESCRT proteins operate at different biological locations and mechanisms of their recruitment are highly adaptable and so might be their mechanisms of scission.

\section{ESCRTs in plasma membrane repair}


The ability of eukaryotic cells to reseal damaged membranes within a few seconds is essential for their viability. The plasma membrane (PM) can be damaged by a number of different stressors such as mechanical rupture, pathological conditions and various toxins that can form holes of different sizes and shapes. Maintaining PM integrity is crucial for cell homeostasis and survival. Calcium influx through small PM lesions $(<100 \mathrm{~nm})$ triggers ESCRT recruitment and their involvement in a repair mechanism. Interestingly, neither ESCRT-0, ESCRT-II nor CHMP-6 have been identified as recruiters of ESCRT-III complex at the sites of PM damage. Instead, together with the EF-hand calcium sensor ALG-2, ALIX is the key recruiter of TSG101 and ESCRT-III machinery [30, 31]. At the site of damage, other ESCRT subunits that appear to colocalize with CHMP4B-positive spots are the ESCRT-III subunits CHMP3, CHMP2A, and CHMP2B, and the ESCRT-III-related CHMP1A, with maximum levels of CHMP4B observed at the wound closure [30, 31]. Keeping in mind the topology of the ESCRT machinery, it is reasonable to assume that it works by promoting extracellular budding of the damaged membrane area, thereby coordinating wound removal with abscission of an extracellular vesicle (Fig. 1C). ESCRT recruitment is rapid and happens as early as 30 seconds to 4 minutes after injury, followed by the repair of the wound within minutes after damage. Whereas recruitment of CHMP4B to the sites of damage is energy independent, both membrane repair and ESCRT-mediated shedding are energy dependent [30]. In addition, the ATPase responsible for the disassembly of ESCRT-III polymers, VPS4, is also employed to the sites of the damage. Another mechanism of PM repair triggered by elevated levels of intracellular calcium is mediated by lysosomal fusion with the PM. Here, the lysosomal enzyme acid sphingomyelinase triggers loss of PM tension by inserting into it [32-34].

\section{ESCRTs in nuclear envelope sealing and repair}

The nuclear envelope (NE) is a bilayered membrane that separates chromatin from the cytoplasm. Besides providing nucleo-cytoplasmic compartmentalization, the NE physically protects the cell's genome integrity. Remodeling of the NE is required for cell growth, division and differentiation, and if perturbed can lead to devastating diseases [35]. Studies on NE reformation in late anaphase have established an essential role of the ESCRT machinery in the closure of remaining gaps in the NE [5, 36, 37]. Here, the ESCRT-II and -III-related protein CHMP7 recruits ESCRT-III to assemble at the microtubule-traversed holes of the growing NE. Together with the ATPase Spastin, ESCRT-III and VPS4 promote NE sealing and microtubule severing [5]. A recent study has uncovered that LEM (LAP2, emerin, MAN1) domain proteins, which are found in the inner NE, can recruit CHMP7 during NE reformation [38]. This suggests that ESCRT-III proteins may enter the nucleoplasm in order to initiate membrane sealing.

Interestingly, ESCRT-dependent repair mechanisms of interphase NE lesions that arise upon nuclear deformation in migrating cells appear to be parallel in major parts to their role in NE sealing upon mitotic exit $[5,36,39,40]$. Like with NE sealing during mitotic exit, CHMP7 is the key initiator of CHMP4B filament assembly upon NE damage, which ultimately drives nuclear membrane repair [38]. For long it has been assumed that a ruptured NE would have lethal consequences for the cell as cytoplasmic nucleases that normally protect cells from foreign DNA could potentially enter the nucleus and damage the endogenous genome [41]. However, in recent years it has been shown that during interphase transient NE rupture 
allows exchange of the molecules between the cytoplasm and the nucleus [42-45]. In addition, it has been shown that cells are able to overcome and restore NE integrity even after repeated NE rupture [39, 40]. The exact mechanism by which the ESCRT-III machinery and VPS4 function in mediating NE repair remains largely elusive. A possible model could be that due to NE rupture, highly unstable exposed hydrophilic domains of the inner- and outernuclear membrane fuse to each other and create a channel connecting cytoplasm and nuclear interior. An interaction of the LEM-domain proteins and CHMP7 at the sites of fusion could further recruit CHMP4B to assemble into a filament (Fig. 1B). The polymerized ESCRT-III subunits containing CHMP2A could in turn recruit the ATPase VPS4B. Finally, tightening of the ESCRT-III filaments would further lead to channel closure and eventually membrane scission.

\section{Conclusions and Perspectives}

The ESCRT machinery has emerged as a central player in sealing of two very different membranes, the PM and the NE. In spite of the differences, the two ESCRT-mediated sealing processes are likely to be mechanistically related. If we consider the canonical function of ESCRTs in ILV formation in endosomes (Fig. 1A), the topology of sealing of the double membrane of the NE would be similar (Fig. 1B), as would the topology of ESCRTmediated shedding of PM vesicles that contain lesions (Fig. 1C). However, the latter mechanisms still have to be experimentally verified.

Concerning other cellular membranes, we know that endolysosomal membranes in particular can be damaged by a number of different compounds such as reactive oxygen species (ROS), amphiphilic drugs, bacterial toxins or cholesterol crystals. Therefore, it is reasonable to assume that cellular mechanisms must exist for the repair of damaged endosomes and lysosomes. What is known so far is that damaged organelles are engulfed and degraded by autophagy when severely damaged, but very little is known about what happens to these organelles when containing only minor lesions in their membranes. Very recently, it has been shown that during limited damage of the lysosome membrane, the ESCRT machinery is recruited to repair the damage prior to eventual engagement of the autophagic machinery [46]. Thus, lysosome repair can be added to the list of ESCRT-mediated membrane repair processes, and it will be interesting to learn about the physiological importance of this novel repair mechanism.

\section{Acknowledgements:}

MR is a postdoctoral fellow of the South-Eastern Norway Regional Health Authority. HS is supported by the Norwegian Cancer Society. This work was partly supported by the Research Council of Norway through its Centres of Excellence funding scheme, project number 262652. 


\section{Figure legend}

Figure 1: Schematic representation of ESCRT-mediated sealing processes

A) In MVB biogenesis, ESCRT-0 initiates the process by capturing ubiquitinated cargo in the endosome membrane and recruiting ESCRT-I, -II and -III.

B) During nuclear envelope sealing, interaction of CHMP7 and LEM-domain proteins results in recruitment of the ESCRT-III complex.

C) ESCRT-mediated shedding of plasma membrane vesicles containing lesions. Here, ESCRT-III is recruted by ALG-2/ALIX and the ESCRT-I complex.

Abbreviations: MVB, multivesicular body; ILV, intraluminal vesicles; ONM, outer nuclear membrane; INM, inner nuclear membrane. 


\section{References}

1. Katzmann, D. J., Babst, M. \& Emr, S. D. (2001) Ubiquitin-dependent sorting into the multivesicular body pathway requires the function of a conserved endosomal protein sorting complex, ESCRT-I, Cell. 106, 145-55.

2. Christopher K. Raymond, I. H.-S., Carol A. Vater, and Tom H. Stevens (1992) Morphological Classification of the Yeast Vacuolar Protein Sorting Mutants: Evidence for a Prevacuolar Compartment in Class E vps Mutants, Molecular biology of the cell. 3, 1389-1402.

3. Raiborg, C. \& Stenmark, H. (2009) The ESCRT machinery in endosomal sorting of ubiquitylated membrane proteins, Nature. 458, 445-52.

4. Carlton, J. G. \& Martin-Serrano, J. (2007) Parallels between cytokinesis and retroviral budding: a role for the ESCRT machinery, Science (New York, NY). 316, 1908-12.

5. Vietri, M., Schink, K. O., Campsteijn, C., Wegner, C. S., Schultz, S. W., Christ, L., Thoresen, S. B., Brech, A., Raiborg, C. \& Stenmark, H. (2015) Spastin and ESCRT-III coordinate mitotic spindle disassembly and nuclear envelope sealing, Nature. 522, 231-5.

6. Christ, L., Wenzel, E. M., Liestol, K., Raiborg, C., Campsteijn, C. \& Stenmark, H. (2016) ALIX and ESCRT-I/II function as parallel ESCRT-III recruiters in cytokinetic abscission, The Journal of cell biology. 212, 499-513.

7. VerPlank, L., Bouamr, F., LaGrassa, T. J., Agresta, B., Kikonyogo, A., Leis, J. \& Carter, C. A. (2001) Tsg101, a homologue of ubiquitin-conjugating (E2) enzymes, binds the L domain in HIV type 1 Pr55(Gag), Proceedings of the National Academy of Sciences of the United States of America. 98, 7724-9.

8. Jennifer E. Garrus, U. K. v. S., Owen W. Pornillos, Scott G. Morham, Kenton H. Zavitz, Hubert E. Wang, Daniel A. Wettstein, Kirsten M. Stray, Melanie Cote, Rebecca L. Rich, David G. Myszka, and Wesley I. Sundquist (2001) Tsg101 and the vacuolar protein sorting pathway are essential for HIV-1 budding, Cell. 107, 55-65.

9. Martin-Serrano, J., Zang, T. \& Bieniasz, P. D. (2001) HIV-1 and Ebola virus encode small peptide motifs that recruit Tsg101 to sites of particle assembly to facilitate egress, Nature medicine. 7, 13139.

10. Demirov, D. G., Ono, A., Orenstein, J. M., Freed, E. O. (2002) Overexpression of the N-terminal domain of TSG101 inhibits HIV-1 budding by blocking late domain function, Proceedings of the National Academy of Sciences of the United States of America. 99, 955-60.

11. Gaullier, J. M., Simonsen, A., D'Arrigo, A., Bremnes, B., Stenmark, H. \& Aasland, R. (1998) FYVE fingers bind PtdIns(3)P, Nature. 394, 432-3.

12. Burd, C. G. \& Emr, S. D. (1998) Phosphatidylinositol(3)-phosphate signaling mediated by specific binding to RING FYVE domains, Molecular cell. 2, 157-62.

13. Raiborg, C., Bremnes, B., Mehlum, A., Gillooly, D. J., D'Arrigo, A., Stang, E. \& Stenmark, H. (2001) FYVE and coiled-coil domains determine the specific localisation of Hrs to early endosomes, Journal of cell science. 114, 2255-63. 
14. Bache, K. G., Raiborg, C., Mehlum, A. \& Stenmark, H. (2003) STAM and Hrs are subunits of a multivalent ubiquitin-binding complex on early endosomes, The Journal of biological chemistry. 278, 12513-21.

15. Alonso, Y. A. M., Migliano, S. M. \& Teis, D. (2016) ESCRT-III and Vps4: a dynamic multipurpose tool for membrane budding and scission, FEBS J. 283, 3288-302.

16. Henne, W. M., Buchkovich, N. J. \& Emr, S. D. (2011) The ESCRT pathway, Developmental cell. 21, 77-91.

17. McCullough, J., Colf, L. A. \& Sundquist, W. I. (2013) Membrane fission reactions of the mammalian ESCRT pathway, Annual review of biochemistry. 82, 663-92.

18. Hurley, J. H. \& Hanson, P. I. (2010) Membrane budding and scission by the ESCRT machinery: it's all in the neck, Nature reviews Molecular cell biology. 11, 556-66.

19. Dobrowolski, R. \& De Robertis, E. M. (2011) Endocytic control of growth factor signalling: multivesicular bodies as signalling organelles, Nature reviews Molecular cell biology. 13, 53-60.

20. Natalie Elia, R. S., Tighe A. Spurlin, James H. Hurley, and Jennifer Lippincott-Schwartz (2011) Dynamics of endosomal sorting complex required for transport (ESCRT) machinery during cytokinesis and its role in abscission, Proceedings of the National Academy of Sciences of the United States of America. 108, 4846-4851.

21. Julien Guizetti, L. S., Jana Mäntler, Sandra Maar, Ina Poser, \& Heinrich Leonhardt, T. M.-R., Daniel W. Gerlich (2011) Cortical constriction during abscission involves helices of ESCRT-III-dependent filaments, Science (New York, NY). 331, 1616-1620.

22. Schoneberg, J., Lee, I. H., Iwasa, J. H. \& Hurley, J. H. (2017) Reverse-topology membrane scission by the ESCRT proteins, Nat Rev Mol Cell Biol. 18, 5-17.

23. Shen, Q. T., Schuh, A. L., Zheng, Y., Quinney, K., Wang, L., Hanna, M., Mitchell, J. C., Otegui, M. S., Ahlquist, P., Cui, Q. \& Audhya, A. (2014) Structural analysis and modeling reveals new mechanisms governing ESCRT-III spiral filament assembly, The Journal of cell biology. 206, 763-77.

24. Suman Lata, G. S., Ankur Jain, Ricardo Pires, Jacob Piehler, \& Heinrich G. Gottlinger, W. W. (2008) Helical Structures of ESCRT-III Are Disassembled by VPS4, Science (New York, NY). 321, 1354-1357.

25. II-Hyung Lee, H. K., Lars-Anders Carlson, Jay T. Groves, and James H. Hurley Negative membrane curvature catalyzes nucleation of endosomal sorting complex required for transport (ESCRT)-III assembly, Proceedings of the National Academy of Sciences of the United States of America. 112, 15892-15897.

26. Chiaruttini, N., Redondo-Morata, L., Colom, A., Humbert, F., Lenz, M., Scheuring, S. \& Roux, A. (2015) Relaxation of Loaded ESCRT-III Spiral Springs Drives Membrane Deformation, Cell. 163, 86679.

27. Peel, S., Macheboeuf, P., Martinelli, N. \& Weissenhorn, W. (2011) Divergent pathways lead to ESCRT-III-catalyzed membrane fission, Trends Biochem Sci. 36, 199-210.

28. Baumgartel, V., Ivanchenko, S., Dupont, A., Sergeev, M., Wiseman, P. W., Krausslich, H. G., Brauchle, C., Muller, B. \& Lamb, D. C. (2011) Live-cell visualization of dynamics of HIV budding site interactions with an ESCRT component, Nature cell biology. 13, 469-74. 
29. Fabrikant, G., Lata, S., Riches, J. D., Briggs, J. A., Weissenhorn, W. \& Kozlov, M. M. (2009) Computational model of membrane fission catalyzed by ESCRT-III, PLoS Comput Biol. 5, e1000575.

30. Jimenez, A. J., Maiuri, P., Lafaurie-Janvore, J., Divoux, S., Piel, M. \& Perez, F. (2014) ESCRT machinery is required for plasma membrane repair, Science (New York, NY). 343, 1247136.

31. Scheffer, L. L., Sreetama, S. C., Sharma, N., Medikayala, S., Brown, K. J., Defour, A. \& Jaiswal, J. K. (2014) Mechanism of $\mathrm{Ca}(2)(+)$-triggered ESCRT assembly and regulation of cell membrane repair, Nat Commun. 5, 5646.

32. Tam, C., Idone, V., Devlin, C., Fernandes, M. C., Flannery, A., He, X., Schuchman, E., Tabas, I. \& Andrews, N. W. (2010) Exocytosis of acid sphingomyelinase by wounded cells promotes endocytosis and plasma membrane repair, The Journal of cell biology. 189, 1027-38.

33. Andrews, N. W., Almeida, P. E. \& Corrotte, M. (2014) Damage control: cellular mechanisms of plasma membrane repair, Trends in cell biology. 24, 734-42.

34. Defour, A., Van der Meulen, J. H., Bhat, R., Bigot, A., Bashir, R., Nagaraju, K. \& Jaiswal, J. K. (2014) Dysferlin regulates cell membrane repair by facilitating injury-triggered acid sphingomyelinase secretion, Cell death \& disease. 5, e1306.

35. Ungricht, R. \& Kutay, U. (2017) Mechanisms and functions of nuclear envelope remodelling, Nature reviews Molecular cell biology. 18, 229-245.

36. Olmos, Y., Hodgson, L., Mantell, J., Verkade, P. \& Carlton, J. G. (2015) ESCRT-III controls nuclear envelope reformation, Nature. 522, 236-9.

37. Olmos, Y., Perdrix-Rosell, A. \& Carlton, J. G. (2016) Membrane Binding by CHMP7 Coordinates ESCRT-III-Dependent Nuclear Envelope Reformation, Current biology : CB. 26, 2635-2641.

38. Mingyu Gu, D. L., Opal S. Chen, Alexander von Appen, Mark S. Ladinsky, Michael J. Redd, Linda Nikolova, Pamela J. Bjorkman, Wesley I. Sundquist, Katharine S. Ullmanc, and Adam Frost (2017) LEM2 recruits CHMP7 for ESCRT-mediated nuclear envelope closure in fission yeast and human cells, Proceedings of the National Academy of Sciences of the United States of America, E2166-E2175.

39. Matthew Raab, M. G., Henry de Belly, Hawa-Racine Thiam, \& Pablo Vargas, A. J. J., Franziska Lautenschlaeger, Raphaël Voituriez, Ana-Maria Lennon-Duménil, Nicolas Manel, Matthieu Piel (2016) ESCRT III repairs nuclear envelope ruptures during cell migration to limit DNA damage and cell death, Science (New York, NY). 352, 359-362.

40. Celine M. Denais, R. M. G., Philipp Isermann, Alexandra L. McGregor,, Mariska te Lindert, B. W., Patricia M. Davidson, Peter Friedl, \& Katarina Wolf, J. L. (2016) Nuclear envelope rupture and repair during cancer cell migration, Science (New York, NY). 352, 353-358.

41. Lammerding, P. I. J. (2017) Consequences of a tight squeeze: Nuclear envelope rupture and repair, Nucleus. 8, 1-7.

42. De Vos, W. H., Houben, F., Kamps, M., Malhas, A., Verheyen, F., Cox, J., Manders, E. M., Verstraeten, V. L., van Steensel, M. A., Marcelis, C. L., van den Wijngaard, A., Vaux, D. J., Ramaekers, F. C. \& Broers, J. L. (2011) Repetitive disruptions of the nuclear envelope invoke temporary loss of cellular compartmentalization in laminopathies, Hum Mol Genet. 20, 4175-86. 
43. Vargas, J. D., Hatch, E. M., Anderson, D. J. \& Hetzer, M. W. (2012) Transient nuclear envelope rupturing during interphase in human cancer cells, Nucleus. 3, 88-100.

44. Hatch, E. M. \& Hetzer, M. W. (2016) Nuclear envelope rupture is induced by actin-based nucleus confinement, The Journal of cell biology. 215, 27-36.

45. Robijns, J., Molenberghs, F., Sieprath, T., Corne, T. D., Verschuuren, M. \& De Vos, W. H. (2016) In silico synchronization reveals regulators of nuclear ruptures in lamin A/C deficient model cells, Scientific reports. 6, 30325.

46. Skowyra, M. L., Schlesinger, P. H., Naismith, T. V. \& Hanson, P. I. (2018) Triggered recruitment of ESCRT machinery promotes endolysosomal repair, Science. 360. 


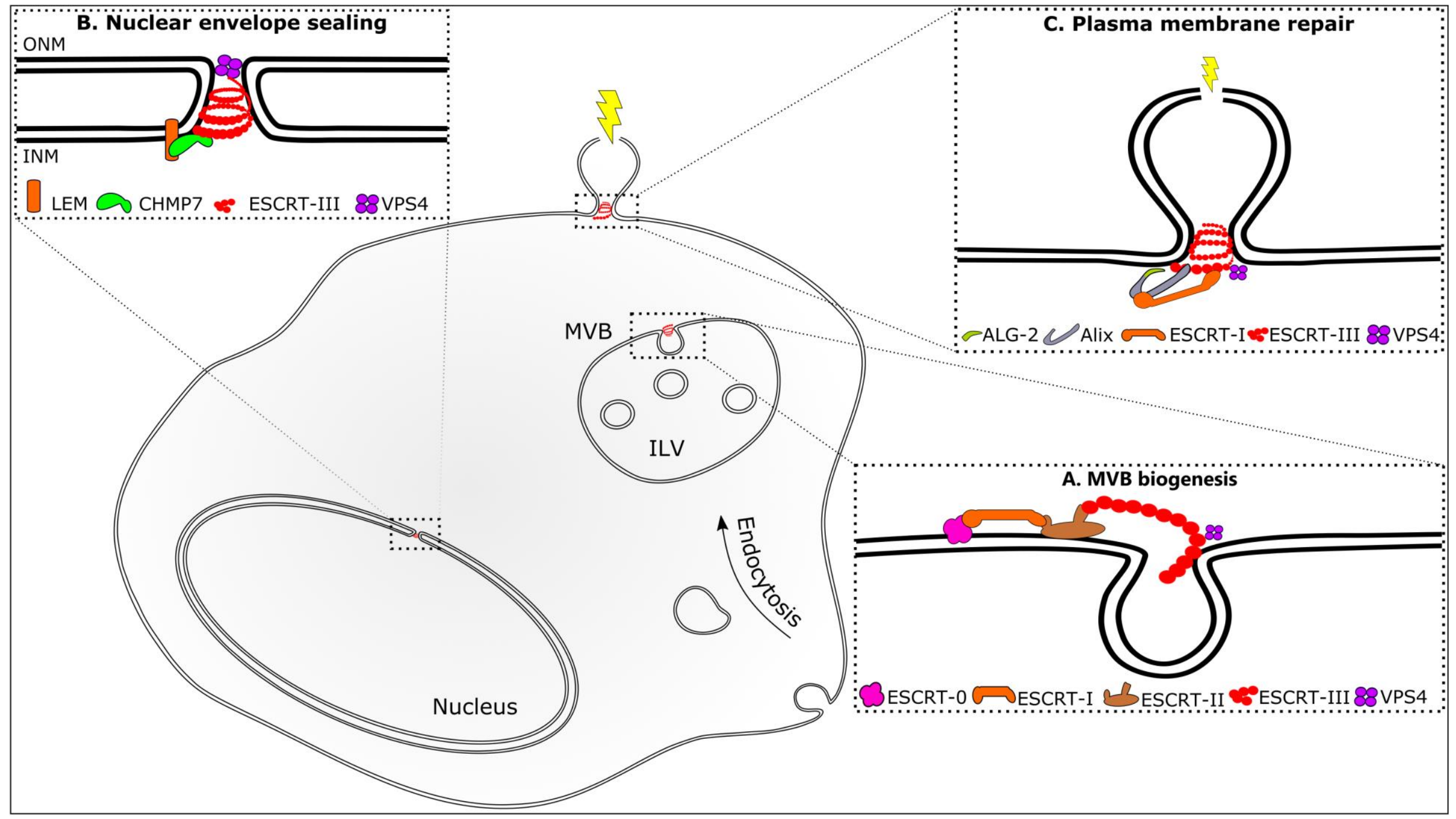

